\title{
Clinical dose profile of Gamma Knife stereotactic radiosurgery for extensive brain metastases
}

\author{
Gregory Neil Bowden, MD, Jong Oh Kim, PhD, ${ }^{2}$ Andrew Faramand, MD, ${ }^{3}$ Kevin Fallon, MS, ${ }^{4}$ \\ John Flickinger, MD, ${ }^{2}$ and L. Dade Lunsford, MD $^{3}$

\begin{abstract}
'Department of Neurological Surgery, University of Alberta, Edmonton, Alberta, Canada; Departments of ${ }^{2}$ Radiation Oncology (Medical Physics) and ${ }^{3}$ Neurological Surgery and the Center for Image-Guided Neurosurgery, University of Pittsburgh, Pennsylvania; and ${ }^{4}$ Department of Radiation Oncology (Medical Physics), Medical University of South Carolina, Charleston, South Carolina
\end{abstract}

OBJECTIVE The use of Gamma Knife stereotactic radiosurgery (GKSRS) for the treatment of extensive intracranial metastases has been expanding due to its superior dosimetry and efficacy. However, there remains a dearth of data regarding the dose parameters in actual clinical scenarios. The authors endeavored to calculate the radiation dose to the brain when treating $\geq 15$ brain metastases with GKSRS.

METHODS This retrospective analysis reviewed dosage characteristics for patients requiring single-session GKSRS for the treatment of $\geq 15$ brain metastases. Forty-two patients met the inclusion criteria between 2008 and 2017. The median number of tumors at the initial GKSRS procedure was 20 (range 15-39 tumors), accounting for 865 tumors in this study. The median aggregate tumor volume was $3.1 \mathrm{~cm}^{3}$ (range $0.13-13.26 \mathrm{~cm}^{3}$ ), and the median marginal dose was 16 Gy (range 14-19 Gy).

RESULTS The median of the mean brain dose was 2.58 Gy (range $0.95-3.67 \mathrm{~Gy}$ ), and $79 \%$ of patients had a dose $<3$ Gy. The 12-Gy dose volume was a median of $12.45 \mathrm{~cm}^{3}$, which was equivalent to $0.9 \%$ of the brain volume. The median percentages of brain receiving $5 \mathrm{~Gy}$ and $3 \mathrm{~Gy}$ were $6.7 \%$ and $20.4 \%$, respectively. There was no correlation between the number of metastases and the mean dose to the brain $(p=0.8)$. A greater tumor volume was significantly associated with an increased mean brain dose $(p<0.001)$. The median of the mean dose to the bilateral hippocampi was $2.3 \mathrm{~Gy}$. Sixteen patients had supplementary GKSRS, resulting in an additional mean dose of $1.4 \mathrm{~Gy}$ (range $0.2-3.8 \mathrm{~Gy}$ ) to the brain.

CONCLUSIONS GKSRS is a viable means of managing extensive brain metastases. This procedure provides a relatively low dose of radiation to the brain, especially when compared with traditional whole-brain radiation protocols.

https://thejns.org/doi/abs/10.3171/2020.3.JNS193369

KEYWORDS Gamma Knife; brain metastases; whole-brain radiation; dose; hippocampus; tumor; oncology; stereotactic radiosurgery

$\mathrm{T}$ HE variables involved in calculating survival from extensive metastatic cancer are invariably numerous and complex, resulting in limitations to our ability to predict long-term survival. ${ }^{1}$ However, disseminated cancer, regardless of tumor origin, is associated with a decrease in survival, especially when intracranial metastases are identified. ${ }^{2}$ The improvements in prognosis with evolving systemic management strategies has made the need for stereotactic radiosurgery (SRS) more critical in decreasing brain-related mortality, tumor progression, and neurological sequalae. ${ }^{3}$ There is now significant evidence supporting the use of Gamma Knife SRS (GKSRS) in much greater numbers of metastases with comparable tumor control and survival. ${ }^{4-6}$

Dosimetry studies consistently demonstrate that GKSRS has the lowest gradient index compared with other SRS modalities..$^{7-10}$ This results in significantly better protection of the normal brain tissue from negative effects of radiation. It also allows GKSRS the potential for treating multiple metastases effectively and safely. Analysis of the literature clearly demonstrates an expanded use of GKSRS in cases of multiple brain metastases. Therefore, it is necessary to determine the radiation dose to the brain in actual cases of diffuse metastases. In this study, we present the 
TABLE 1. Data related to tumor type for initial radiosurgery

\begin{tabular}{lrrrrrr}
\hline & & \multicolumn{4}{c}{ Median (range) } & \\
\cline { 3 - 6 } & $\begin{array}{c}\text { No. of } \\
\text { Pts }\end{array}$ & $\begin{array}{c}\text { Age at SRS, } \\
\text { yrs }\end{array}$ & $\begin{array}{c}\text { No. of Tumors/ } \\
\text { Pt }\end{array}$ & $\begin{array}{c}\text { Margin Dose, } \\
\text { Gy }\end{array}$ & $\begin{array}{c}\text { Aggregate Tumor } \\
\text { Vol, cm }{ }^{3}\end{array}$ & $\begin{array}{c}\text { No. of Pts w/ } \\
\text { Prior WBRT (\%) }\end{array}$ \\
\hline Breast & 9 & $55(35-86)$ & $19(15-26)$ & $15(14-16)$ & $1.5(0.5-6.9)$ & $2(22)$ \\
\hline Melanoma & 11 & $56(28-77)$ & $21(15-35)$ & $16(15-19)$ & $4.0(0.1-9.8)$ & $1(9)$ \\
\hline SCLC & 6 & $59(56-73)$ & $18(15-26)$ & $15(14-16)$ & $1.5(0.4-4.0)$ & $6(100)$ \\
\hline NSCLC & 16 & $64(47-83)$ & $21(15-39)$ & $16(14-18)$ & $2.8(0.2-13.3)$ & $5(31)$ \\
\hline Total & 42 & $60(28-86)$ & $20(15-39)$ & $16(14-19)$ & $3.1(0.1-9.8)$ & $14(33)$ \\
\hline
\end{tabular}

NSCLC = non-SCLC; $p t=$ patient; $S C L C=$ small cell lung cancer.

dose distributions for patients who underwent GKSRS for $\geq 15$ intracranial tumors.

\section{Methods \\ Patient Characteristics}

A single-institution retrospective analysis approved by the University of Pittsburgh institutional review board was conducted. This analysis evaluated the radiation dose to the brain of 42 patients with $\geq 15$ brain metastases from breast cancer ( 9 patients), lung cancer (16 non-small cell lung cancer and 6 small cell lung cancer patients), and melanoma (11 patients) (Table 1). GKSRS was conducted between December 2008 and December 2017. Patient clinical data were collected for a recent publication on extensive metastatic disease. ${ }^{11}$ We subsequently identified a subset of patients treated in a single procedure (not staged) at a consistent margin dose and with no prior craniotomies or radiosurgery. Patients in the study had a significantly greater number of tumors on the day of SRS, refused whole-brain radiation therapy (WBRT), or presented for salvage treatment post-WBRT. A total of 865 tumors were treated during the initial SRS procedures. A median of 20 tumors per patient (range 15-39 tumors) were treated. The median aggregate tumor volume was $3.1 \mathrm{~cm}^{3}$ (range $0.13-13.26 \mathrm{~cm}^{3}$ ), which represented a median treatment volume of $7.2 \mathrm{~cm}^{3}$ (range 1.1-25 $\mathrm{cm}^{3}$ ). The median dose to the margin was 16 Gy (range 14-19 Gy), and the median percent isodose was $80 \%$ (range 50\%-90\%). Fourteen patients received prior WBRT. The mean follow-up time was 12.77 months (range 0-43.5 months).

This paper represents a dose analysis of a recent publication on patients with $\geq 15$ brain metastases that included 93 patients and 3016 tumors. ${ }^{11}$ Patients had a median survival after GKSRS of 16 months for breast cancer, 4.6 months for lung cancer, and 3.1 months for melanoma. ${ }^{11}$ The control rates for breast cancer, lung cancer, and melanoma were $94.9 \%, 97.3 \%$, and 97.5, respectively, but were limited by short survival times in lung cancer and melanoma patients. ${ }^{11}$

\section{Radiosurgery Technique}

The specific details of our GKSRS procedure have been documented in our previous publications. ${ }^{11,12}$ In short, the patients in the study were managed as outpatients. Mild sedation with intravenous midazolam and fentanyl was utilized for frame placement. In addition, a local anesthetic was administered at the pin locations for the
Leksell frame placement. Patients receiving a mask-based procedure in conjunction with the ICON unit (Elekta AB) did not receive pain or sedating medications. High-resolution axial imaging (MRI unless contraindicated) was performed. GammaPlan software (Elekta AB) was utilized to create a conformal plan that incorporated the enhancing tumor volume of all identifiable tumors. Forward planning was performed for all patients. It was necessary to utilize advanced planning techniques to avoid the disruption of adjacent tumor plans. This analysis encompasses procedures using Leksell Gamma Knife units (Elekta AB) Perfexion and ICON models.

Patients receiving SRS were followed every $2-3$ months with clinical and imaging follow-up. Evidence of local or distant disease progression led to a recommendation of an additional SRS procedure when appropriate. Methylprednisolone was administered to all patients at the end of their procedure. Patients with changes in their symptoms between follow-up evaluations were assessed on an urgent basis for tumor growth or potential adverse radiation effects.

To assess the distribution of dose with an extensive metastatic distribution, we determined the median dose to the whole brain. We also determined the volume and percentage of the brain receiving a dose of $12 \mathrm{~Gy}, 5 \mathrm{~Gy}$, and $3 \mathrm{~Gy}$ while accounting for tumor volume. The median brain volume was $1408 \mathrm{~cm}^{3}$ based on anatomical modeling fused onto the treatment MRI scan. Furthermore, the hippocampi were outlined using anatomical modeling to provide accurate dosimetry of these structures and to limit any potential bias from individual freehand drawings of the hippocampal pathway. Technical data regarding the GKSRS procedures were collected from GammaPlan (Elekta) and analyzed retrospectively by physicists and physicians.

\section{Statistical Analysis}

Scatterplots were used to illustrate metastatic number, volume, and radiation dose. The log-rank test was used to determine interactions between radiation dose and related variables. Continuous features were summarized using medians and ranges. Pearson's $\mathrm{r}^{2}$ was used to assess the correlation of volumes and doses between the 2 imaging modalities. A positive Pearson's $r$ indicated a positive correlation between tested variables. Statistical analysis was performed using IBM SPSS Statistics (version 25, IBM Corp.); $\mathrm{p}<0.05$ was set for statistical significance. 


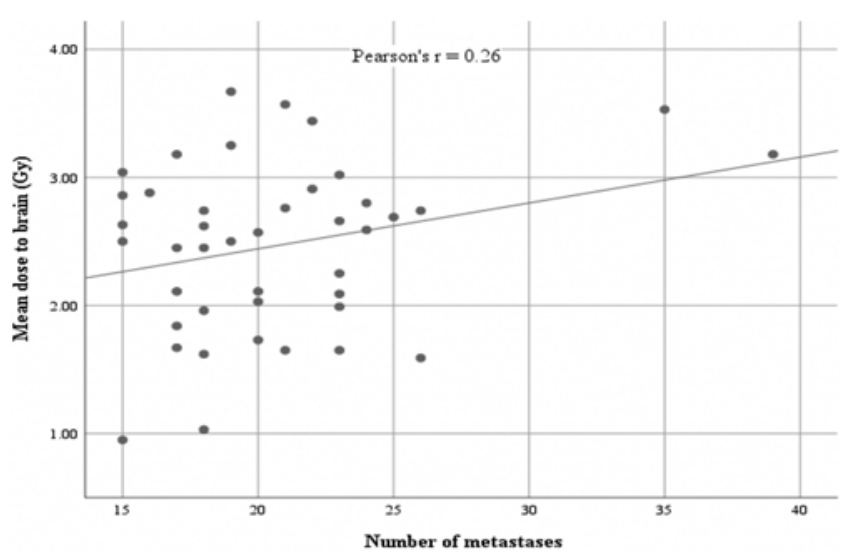

FIG. 1. Graphic representation of the mean dose to the brain versus the number of metastases treated during the initial GKSRS procedure. No significance was identified $(p=0.1)$.

\section{Results}

The median of the mean dose to the brain was 2.58 Gy (range $0.95-3.67 \mathrm{~Gy}$ ), and $79 \%$ of patients had a mean brain dose $<3 \mathrm{~Gy}$. The 12-Gy volume to the brain was a median of $12.45 \mathrm{~cm}^{3}$ (range $2.44-35.51 \mathrm{~cm}^{3}$ ), equivalent to $0.9 \%$ (range $0.21 \%-2.86 \%$ ) of the brain volume. The 5-Gy and 3-Gy median volumes to the brain were 91.04 $\mathrm{cm}^{3}$ (range 12.4-257.65 $\mathrm{cm}^{3}$ ) and $306.33 \mathrm{~cm}^{3}$ (range $43.6-$ $732.63 \mathrm{~cm}^{3}$ ), respectively. This equates to $6.7 \%$ (range $1.1 \%-20.25 \%$ ) of brain receiving a 5-Gy dose and $20.4 \%$ (range $3.86 \%-47.98 \%$ ) of brain receiving a 3-Gy dose. The median volume of brain receiving a dose of radiation equivalent to the treatment dose for each patient was 4.02 $\mathrm{cm}^{3}$ (range $0.66-13.55 \mathrm{~cm}^{3}$ ), which is equivalent to a median of $0.27 \%$ of the brain volume.

There was no correlation between the number of metastases and the volume of treatment $(p=0.8)$. There was also no association between the number of metastases and the mean dose to the brain $(\mathrm{p}=0.8)$ (Fig. 1). The number of metastases was correlated to the volume of brain receiving $3 \mathrm{~Gy}(\mathrm{p}=0.03)$, but it was not associated with the $5-$ Gy or 12-Gy volumes.

A greater tumor volume was significantly associated with a higher mean dose to the brain ( $<$ < 0.001) (Fig. 2). This volume relationship to dose received by the brain was evident across the 12-Gy, 5-Gy, and 3-Gy calculations ( $\mathrm{p}$ $<0.001)$. An aggregate tumor volume $>3 \mathrm{~cm}^{3}$ resulted in a significantly higher mean dose to the brain $(\mathrm{p}<0.001)$. There was no significant difference in volume related to tumor type $(\mathrm{p}=0.4)$. Maximum dose had a significant but borderline correlation with the mean brain dose $(\mathrm{p}=$ 0.03 ). A significant but borderline negative correlation was found between mean dose to the brain and margin dose $(\mathrm{p}$ $=0.04$ ).

The median of the mean dose to the bilateral hippocampi was $2.3 \mathrm{~Gy}$. The median minimum dose was $1 \mathrm{~Gy}$ (range 0-2.7 Gy), and the maximum was 4.7 Gy (range 0.2-30.9 Gy). This includes 10 patients (24\%) who had a tumor in direct contact with one of these structures, which was demonstrated by imaging and a maximum dose greater than the prescribed margin dose. Patients without direct

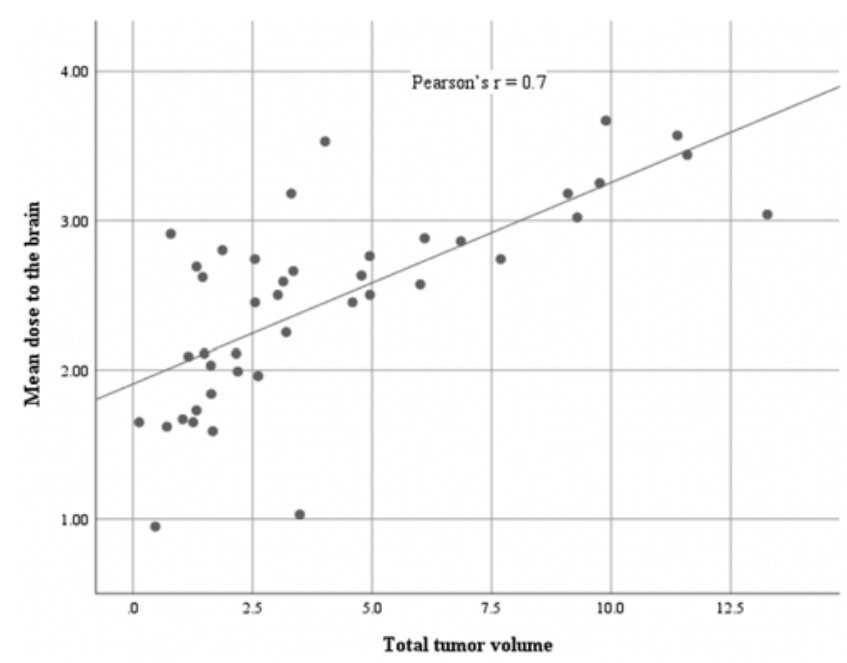

FIG. 2. Graphic representation of the mean dose to the brain versus the tumor volume of the metastases treated during the initial GKRS procedure. A greater volume correlated with an increased mean brain dose ( $p$ $<0.001)$.

tumor contact with the hippocampus had a median dose of $1.7 \mathrm{~Gy}$, which translated to a median maximum dose of $3.8 \mathrm{~Gy}$. An additional $12 \%$ of patients had tumors immediately adjacent to one of the hippocampal structures.

Sixteen patients $(38 \%)$ had additional GKSRS procedures (2-5), accounting for the treatment of an additional 300 tumors. These subsequent procedures resulted in a mean dose of $1.4 \mathrm{~Gy}$ (range $0.2-3.8 \mathrm{~Gy}$ ) to the brain in this subpopulation over time. The tumor control rate was $96.6 \%$. Six patients (14\%) required re-treatment due to local tumor progression. In this subset of patients, the median survival times after GKSRS were 17.5 months, 4.2 months, and 7.83 months for breast cancer, lung cancer, and melanoma, respectively. Four patients (9.5\%) developed leptomeningeal disease: 1 with breast cancer at 22.4 months, 1 with lung cancer at 8.1 months, and 2 with melanoma at a mean of 1 month after SRS.

Fourteen patients had received prior WBRT, of whom 8 patients $(57 \%)$ had evidence of leukoencephalopathy at the time of the initial GKSRS treatment (grade 1, 2 patients; grade 2, 5 patients; and grade 3, 1 patient). ${ }^{21}$ In 27 patients with imaging follow-up beyond 2 months (mean 15.3 months), there were 3 patients (33\%) with prior WBRT+GKSRS procedures who demonstrated new leukoencephalopathy changes (grade 1, 2 patients; and grade 2, 1 patient). These patients had a mean brain dose of 2.29 Gy and all had a secondary SRS procedure. In addition, 2 patients with prior WBRT demonstrated worsening of their leukoencephalopathy from grade 2 to grade 3 . The patients with worsening leukoencephalopathy had a mean brain dose of $2.96 \mathrm{~Gy}$. It is unclear as to whether this represented progression of leukoencephalopathy from WBRT or if SRS promoted further exacerbation. Two patients (11\%) developed leukoencephalopathy (grade 1, 1 patient; and grade 3, 1 patient) after GKSRS procedures alone. One SRS-only patient had 18 metastases treated with a tumor volume of $7.83 \mathrm{~cm}^{3}$ and a median brain dose of 2.62 Gy. The second patient had 21 tumors treated with an ag- 
gregate tumor volume of $2 \mathrm{~cm}^{3}$ and a median brain dose of $1.65 \mathrm{~Gy}$.

\section{Discussion}

Extensive metastatic disease is unfortunately a relatively common occurrence, and the use of SRS for these patients is increasing rapidly due to failure of WBRT, a desire to reduce neurocognitive sequalae, and patient preference. Previously published data have demonstrated that treating 17-25 tumors with a $20-$ Gy margin at $50 \%$ isodose still results in a median dose to the brain of $<5 \mathrm{~Gy}{ }^{13,14}$ The mean margin dose in our current study ranged from 16 to 19 Gy with a median isodose of $80 \%$. The adaptations to planning and technological improvements led to a median dose of $2.58 \mathrm{~Gy}$ in our study when treating 15-39 tumors per patient. The percent dose volumes to the brain for $12 \mathrm{~Gy}, 5 \mathrm{~Gy}$, and $3 \mathrm{~Gy}$ were $0.9 \%, 6.7 \%$, and $20.4 \%$, respectively. This represents a fraction of the dose received by conventional WBRT. Inevitably, the dose distribution becomes more complex according to the increase in the number of targets..$^{15}$ Therefore, planning strategies and a careful assessment of isocenter proximity must be understood in order to avoid interactions between adjacent metastases.

Patients presenting for SRS with an extensive number of brain metastases may still have a total tumor volume that is relatively small, as illustrated by the median aggregate volumes in this study. These small- or intermediate-volume tumors are optimal for GKSRS, which has a superior gradient index and brain dose sparing compared with other modalities. This rapid falloff of dose outside the treatment volume results in reduced 5-Gy and 12-Gy volumes delivered to surrounding normal brain despite a greater central dose. ${ }^{7,10,16}$ In this study, there was no correlation between metastatic tumor number and mean dose to the brain at 12 Gy and 5 Gy, but the 3-Gy volume did marginally rise as the number of metastases increased.

Multiple publications have demonstrated that the total number of metastatic brain tumors is not predictive of tumor control or brain-related survival but is more closely associated with volume. ${ }^{6,17-19}$ The cumulative volume of tumors being treated in this study was significantly correlated to the dose received by the brain. This was evident across all treatment dose volumes analyzed ( $12 \mathrm{~Gy}, 5 \mathrm{~Gy}$, and $3 \mathrm{~Gy}$ ) and reflects the expected distribution of a larger dose of radiation. A negative correlation was demonstrated between mean dose to the brain and margin dose. This is an indication that physicians were willing to treat with a higher margin dose in situations of a smaller overall tumor volume.

Based on a recent survey, the majority of American radiation oncologists are preferentially treating up to 10 brain metastases with SRS, if not more. ${ }^{20}$ Before the widespread use of SRS, many centers typically used WBRT at a standard dose of $30 \mathrm{~Gy}$ in 10 fractions for patients with metastatic cancer regardless of the number of metastases. The impetus for a substantially lower brain dose is reflected in the well-known significant neurocognitive effects of WBRT and an increased risk of leukoencephalopathy. ${ }^{21}$ There have been recent attempts to protect memory function with hippocampal dose sparing. This protocol, as outlined by RTOG 0933 , has a dose to $100 \%$ of the hippocampus of $\leq 9$ Gy and a maximal dose of $\leq 16 \mathrm{~Gy} .{ }^{22}$ The results of our study clearly demonstrate a much lower dose to the hippocampal structures of $1.7 \mathrm{~Gy}$ and a median maximum dose of 3.8 Gy in patients who would be potential candidates for hippocampal-sparing protocols. It should be noted in this study that almost $25 \%$ of patients had tumors either in or adjacent to the hippocampus.

This study demonstrated a high incidence rate of leukoencephalopathy with prior WBRT (79\%) and a low rate with GKSRS being administered alone. This is well documented in the literature, where rates as high as $97 \%$ for WBRT+SRS versus 3\% for SRS alone have been reported. ${ }^{21,23}$ Our data document an extreme scenario of a median of 20 brain metastases but still resulted in $79 \%$ of patients being treated with a mean brain dose $<3 \mathrm{~Gy}$. This is of interest, as doses $>3$ Gy have been demonstrated to increase the risk of leukoencephalopathy. ${ }^{24}$ In addition, 16 patients (38\%) had additional GKSRS procedures for 300 tumors. In this subpopulation, the mean dose to the brain was $1.4 \mathrm{~Gy}$. The option of repeat GKSRS for distant or local disease progression appears to be viable based on brain dose.

\section{Conclusions}

Extensive intracranial metastases are not uncommon, and the use of GKSRS in these scenarios has been increasing rapidly. This study demonstrates that a favorable dose distribution is delivered to patients who undergo GKSRS. This allows neurosurgeons and radiation oncologists in a single outpatient treatment to effectively treat patients with multiple brain metastases while delivering a low dose to the surrounding brain.

\section{References}

1. Kondziolka D, Parry PV, Lunsford LD, et al. The accuracy of predicting survival in individual patients with cancer. $J$ Neurosurg. 2014;120(1):24-30.

2. Gaspar L, Scott C, Rotman M, et al. Recursive partitioning analysis (RPA) of prognostic factors in three Radiation Therapy Oncology Group (RTOG) brain metastases trials. Int J Radiat Oncol Biol Phys. 1997;37(4):745-751.

3. Higuchi Y, Yamamoto M, Serizawa T, et al. Modern management for brain metastasis patients using stereotactic radiosurgery: literature review and the authors' Gamma Knife treatment experiences. Cancer Manag Res. 2018;10:1889-1899.

4. Hughes RT, Masters AH, McTyre ER, et al. Initial SRS for patients with 5 to 15 brain metastases: results of a multiinstitutional experience. Int J Radiat Oncol Biol Phys. 2019;104(5):1091-1098.

5. Serizawa T, Yamamoto M, Higuchi Y, et al. Local tumor progression treated with Gamma Knife radiosurgery: differences between patients with 2-4 versus 5-10 brain metastases based on an update of a multi-institutional prospective observational study (JLGK0901). J Neurosurg. 2020;132(5):14801489.

6. Yamamoto M, Kawabe T, Sato Y, et al. Stereotactic radiosurgery for patients with multiple brain metastases: a casematched study comparing treatment results for patients with 2-9 versus 10 or more tumors. J Neurosurg. 2014;121(suppl):16-25.

7. Cao H, Xiao Z, Zhang Y, et al. Dosimetric comparisons of 
different hypofractionated stereotactic radiotherapy techniques in treating intracranial tumors $>3 \mathrm{~cm}$ in longest diameter. J Neurosurg. 2020;132(4):1024-1032.

8. Dong P, Pérez-Andújar A, Pinnaduwage D, et al. Dosimetric characterization of hypofractionated Gamma Knife radiosurgery of large or complex brain tumors versus linear accelerator-based treatments. J Neurosurg. 2016;125(suppl 1):97-103.

9. Han EY, Wang H, Luo D, et al. Dosimetric comparison of fractionated radiosurgery plans using frameless Gamma Knife ICON and CyberKnife systems with linear accelerator-based radiosurgery plans for multiple large brain metastases. J Neurosurg. 2020;132(5):1473-1479.

10. McDonald D, Schuler J, Takacs I, et al. Comparison of radiation dose spillage from the Gamma Knife Perfexion with that from volumetric modulated arc radiosurgery during treatment of multiple brain metastases in a single fraction. $J \mathrm{Neu}$ rosurg. 2014;121(suppl):51-59.

11. Bowden G, Faramand A, Niranjan A, et al. Gamma Knife radiosurgery for the management of more than 15 cerebral metastases. World Neurosurg. 2019;126:e989-e997.

12. Bowden G, Kano H, Caparosa E, et al. Gamma knife radiosurgery for the management of cerebral metastases from non-small cell lung cancer. J Neurosurg. 2015;122(4):766772.

13. Yamamoto M, Ide M, Nishio Si, Urakawa Y. Gamma Knife radiosurgery for numerous brain metastases: is this a safe treatment? Int J Radiat Oncol Biol Phys. 2002;53(5):1279_1283.

14. Yang CC, Ting J, Wu X, Markoe A. Dose volume histogram analysis of the Gamma Knife radiosurgery treating twentyfive metastatic intracranial tumors. Stereotact Funct Neurosurg. 1998;70(suppl 1):41-49.

15. Takahashi M, Narabayashi I, Kuroiwa T, et al. Stereotactic radiosurgery (SRS) for multiple metastatic brain tumors: effects of the number of target tumors on exposure dose in normal brain tissues. Int J Clin Oncol. 2003;8(5):289-296.

16. Vergalasova I, Liu H, Alonso-Basanta M, et al. Multi-institutional dosimetric evaluation of modern day stereotactic radiosurgery (SRS) treatment options for multiple brain metastases. Front Oncol. 2019;9:483.

17. Grandhi R, Kondziolka D, Panczykowski D, et al. Stereotactic radiosurgery using the Leksell Gamma Knife Perfexion unit in the management of patients with 10 or more brain metastases. J Neurosurg. 2012;117(2):237-245.

18. Hughes RT, McTyre ER, LeCompte M, et al. Clinical outcomes of upfront stereotactic radiosurgery alone for patients with 5 to 15 brain metastases. Neurosurgery. 2019;85(2):257263.
19. Routman DM, Bian SX, Diao K, et al. The growing importance of lesion volume as a prognostic factor in patients with multiple brain metastases treated with stereotactic radiosurgery. Cancer Med. 2018;7(3):757-764.

20. Blomain ES, Kim H, Garg S, et al. Stereotactic radiosurgery practice patterns for brain metastases in the United States: a national survey. J Radiat Oncol. 2018;7(3):241-246.

21. Monaco EA III, Faraji AH, Berkowitz O, et al. Leukoencephalopathy after whole-brain radiation therapy plus radiosurgery versus radiosurgery alone for metastatic lung cancer. Cancer. 2013;119(1):226-232.

22. Gondi V, Pugh SL, Tome WA, et al. Preservation of memory with conformal avoidance of the hippocampal neural stemcell compartment during whole-brain radiotherapy for brain metastases (RTOG 0933): a phase II multi-institutional trial. $J$ Clin Oncol. 2014;32(34):3810-3816.

23. Trifiletti DM, Lee CC, Schlesinger D, et al. Leukoencephalopathy after stereotactic radiosurgery for brain metastases. Int J Radiat Oncol Biol Phys. 2015;93(4):870-878.

24. Cohen-Inbar O, Melmer P, Lee CC, et al. Leukoencephalopathy in long term brain metastases survivors treated with radiosurgery. J Neurooncol. 2016;126(2):289-298.

\section{Disclosures}

Dr. Lunsford is a consultant for Elekta AB, Insightec, and DSMB and a stockholder in Elekta AB.

\section{Author Contributions}

Conception and design: Bowden, Kim, Fallon, Flickinger. Acquisition of data: Bowden, Kim, Fallon. Analysis and interpretation of data: Kim, Faramand, Flickinger. Drafting the article: Bowden. Critically revising the article: Flickinger, Lunsford. Reviewed submitted version of manuscript: all authors. Approved the final version of the manuscript on behalf of all authors: Bowden. Statistical analysis: Faramand. Study supervision: Lunsford.

\section{Correspondence}

Gregory Neil Bowden: University of Alberta, Edmonton, AB, Canada.gregory.bowden@ahs.ca. 